\title{
Histone deacetylase 3 represses HTLV-1 tax transcription
}

\author{
RAUL VILLANUEVA ${ }^{1}$, ANTONIO H. IGLESIAS ${ }^{1}$, SANDRA CAMELO ${ }^{1}$, \\ LUIS C. SANIN ${ }^{1}$, STEVEN G. GRAY ${ }^{1,2}$ and FERNANDO DANGOND ${ }^{1}$ \\ ${ }^{1}$ Laboratory of Transcriptional and Immune Regulation, Brigham and Women's Hospital Laboratories, \\ 65 Landsdowne Street, Cambridge, MA 02139, USA; ${ }^{2}$ Departments of Clinical Medicine and Oncology, \\ Institute of Molecular Medicine, Trinity Sciences Health Centre, St James' Hospital, Dublin 8, Ireland
}

Received January 25, 2006; Accepted March 21, 2006

\begin{abstract}
We examined the epigenetic mechanisms involved in human T-cell lymphotropic virus type 1 (HTLV-1) Tax expression. Blockade of histone deacetylation with trichostatin A (TSA) resulted in Tax upregulation. Using a chromatin immunoprecipitation (ChIP) assay, we verified local histone hyperacetylation at the HTLV-1 LTR in response to TSA. In agreement, HDAC3 transfection led to reductions in both Tax expression and histone acetylation. HDAC3 mutations and deletions spanning the catalytic site had variable ability to repress Tax, but HDAC activity was not essential for repression. Immunoprecipitation studies revealed that Tax co-exists in a complex containing both histone deacetylase 1 (HDAC1) and 3 (HDAC3). Our results suggest that HDACs may actively participate in the repression of HTLV-1 Tax transcription.
\end{abstract}

\section{Introduction}

The HTLV-1 viral Tax protein has been proposed to play crucial roles in the pathogenesis of autoimmune disorders and cancer $(1,2)$. Transformation of T-cells is characterized by IL-2-independent growth (3), a phenomenon in which Tax (4) and JAK/STAT pathway proteins $(5,6)$ have been implicated. In addition, Tax may also play a role in the phenomenon of T-cell spontaneous proliferation, observed in

Correspondence to: Dr Steven G. Gray, Thoracic Oncology Research Group, Departments of Clinical Medicine and Oncology, Institute of Molecular Medicine, Trinity Sciences Health Centre, St. James' Hospital, Dublin 8, Ireland

E-mail: sgray@stjames.ie

Dr Fernando Dangond, Berlex Laboratories, 6 West Belt, Wayne, NJ 07470-6806, USA

E-mail: fernando_dangond@berlex.com

Key words: viral, gene regulation, $\mathrm{t}$ lymphocytes, human T-cell lymphotropic virus type 1
HTLV-1-infected individuals (7). It is believed that some of these effects depend on the ability of Tax to deregulate expression of hosT-cell genes including cytokine and proliferation-promoting genes (8).

The Tax protein itself directly stimulates expression of its own mRNA by binding DNA $(9,10)$ at the Tax-response elements (TREs) on the U3 region of the viral LTR (11-13). Tax protein recruits DNA-binding cellular proteins including ATF/CREB, which associates with the CREB-binding protein $(\mathrm{CBP}) / \mathrm{p} 300(14,15)$, in complexes that facilitate access to the TREs. Tax protein also directly binds the transcriptional coactivators, $\mathrm{CBP} / \mathrm{p} 300$ (16-20) and P/CAF, (21), both proteins containing intrinsic histone acetyltransferase (HAT) activity (22). It has been shown that HAT enzymes lead to histone acetylation and promote transcription in part by relaxing specific nucleosomal-DNA interactions, facilitating the binding of transcription factors to their target gene promoters. In contrast, HDAC corepressors promote nucleosomal-DNA condensation, thus limiting the accessibility of transcription factors and downregulating gene expression $(23,24)$. Blockade of HDAC enzymes by the inhibitor drugs butyrate or trichostatin A (TSA), leads to transcriptional activation of multiple genes by de-repression. In this study, we sought to examine whether histone deacetylase enzyme HDAC3 represses HTLV-1 Tax transcription.

\section{Materials and methods}

Cell growth conditions and treatments. HTLV-1-infected C81 cells were grown in complete medium (RPMI supplemented with $10 \%$ FCS, $10 \mathrm{mM}$ Hepes, $2 \mathrm{mM}$ L-glutamine, and $100 \mathrm{U} / 100 \mu \mathrm{g} / \mathrm{ml}$ penicillin/streptomycin). T-cell clones were maintained at a density of $<1 \times 10^{6}$ cells $/ \mathrm{ml}$ and stimulated with the polyclonal activator, PHA $(1 \mu \mathrm{g} / \mathrm{ml})$, and irradiated feeders for continuous growth. All cells were plated on sterile petri dishes or 96-well plates, cultured in complete medium plus $10 \%$ IL-2 containing T-Stim (Collaborative Biomedical Products) and incubated at $37^{\circ} \mathrm{C}$ with $6 \% \mathrm{CO}_{2}$ and $96 \%$ humidity. HTLV-1-infected human Du43 T-cell clones (kindly provided by Per Höllsberg, Harvard University) were treated for $24 \mathrm{~h}$ with $100 \mathrm{nM}$ TSA (Wako Pure Chemical Industries). HTLV-1-infected human Mu16 T-cell clones 


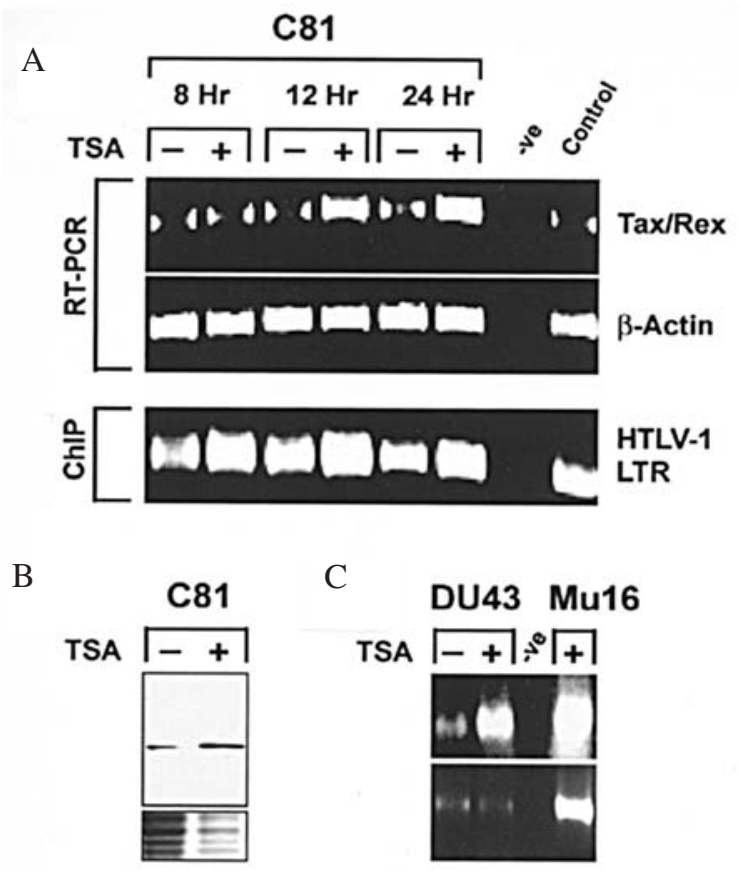

Figure 1. (A) Twelve hours after administration of $100 \mathrm{nM}$ TSA, the mRNA for Tax/Rex is detected by RT-PCR in C81 cells. $\beta$-actin mRNA is shown as a control. Chromatin immunoprecipitation (ChiP) assay (lower panel) shows earlier $(8 \mathrm{~h})$ histone effects: higher PCR amplification of HTLV-1 LTR sequences immunoprecipitated with $\alpha$-acH3 Abs in the TSA-stimulated C81 cells. The persistence of the enhanced Tax mRNA expression at $24 \mathrm{~h}$ correlates with the persistently enhanced genomic acetylation of the LTR region. HUT102 cells are used as control. (B) Treatment of C81 cells with TSA $(10 \mathrm{ng} / \mathrm{ml})$ for $24 \mathrm{~h}$ leads to Tax protein upregulation, as shown by Western blotting. (C) TSA (100 nM) for $24 \mathrm{~h}$ induces higher Tax/Rex mRNA in Du43, an HTLV-1-infected human CD4+ T-cell clone. RNA isolated from Mu16 T-cells, which chronically express higher levels, was used as a Tax-Rex control mRNA. Control cells received vehicle.

(provided by Per Höllsberg) and HUT102 cells, both known to express high levels of Tax, were used as controls.

RNA isolation and RT-PCR. RNA was isolated using the Trizol method (Gibco BRL). We performed RT-PCR with the Retroscript kit (Ambion, Austin, TX) using primers FWD 5'-TGGTCTTAATAGCCGCCAGTGGAA-3', and REV 5'-AGGTGATCTGATGCTCTGGACAGG-3', spanning the second splice junction site of Tax/Rex mRNA (25). We used the $\beta$-actin-specific primers FWD 5'-AACCCCAA GGCCAACCGCGAGAAGATGACC-3', and REV 5'GGTGATGACCTGGCCGTCAGGCAGCTCGTA-3'

PCR conditions included denaturing at $94^{\circ} \mathrm{C}$ for $1 \mathrm{~min}$, annealing at $62^{\circ} \mathrm{C}$ for $1 \mathrm{~min}$, and extension at $72^{\circ} \mathrm{C}$ for $2 \mathrm{~min}$ x 35 or 40 cycles, and a final extension step at $72^{\circ} \mathrm{C}$ for $10 \mathrm{~min}$. The PCR products were electrophoresed in $1 \%$ agarose (Gibco BRL) gels. Bands were detected by ethidium bromide staining.

ChIP assay. For the ChIP assay, cells were treated with $1 \%$ formaldehyde for $10 \mathrm{~min}$ at $37^{\circ} \mathrm{C}$ and collected in lysis buffer [Upstate Biotechnology, Inc (UBI)] containing PMSF, pepstatin A and aprotinin (Sigma). After sonication, lysates were centrifuged, diluted 10-fold in ChIP dilution buffer (UBI),
A

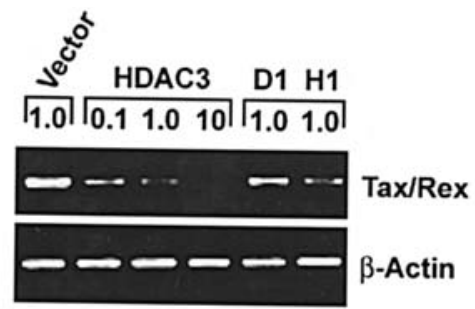

B
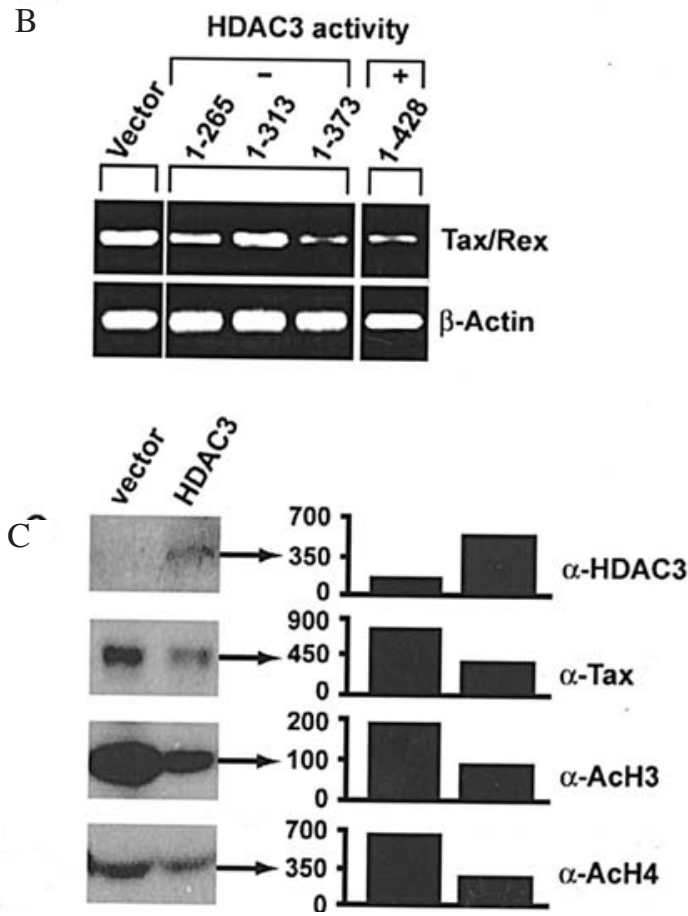

Figure 2. (A) HDAC3 transfection of $\mathrm{C} 81$ cells leads to a dose-dependent $(0.1,1.0$ and $10 \mu \mathrm{g}$ of DNA) decrease in Tax mRNA expression, as shown by RT-PCR. HDAC 3 constructs, D1 and H1, harboring mutations in the enzymatic site (tubular pocket) are less efficient at inducing repression. (B) Transfection of C81 cells with HDAC3 constructs that encode proteins devoid of enzymatic activity. Cells were collected at $48 \mathrm{~h}$ post-transfection for RNA isolation and RT-PCR. The experiment reveals that enzymatic activity is not essential for a repressor function on Tax mRNA expression. (C) Western blotting reveals WT HDAC3 (1-428) transfection for $24 \mathrm{~h}$ that correlates with decreased Tax and lower acetylation of histone proteins. Membranes were incubated with Abs against HDAC3, p40Tax, acH3 and acH4 histones. Densitometry on right panel shows relative units of intensity.

and precleared for $1 \mathrm{~h}$ at $4^{\circ} \mathrm{C}$ with agitation using salmon sperm DNA/Protein A agarose-50\% slurry (UBI). Five $\mu \mathrm{g}$ of $\alpha$ acetylated histone $\mathrm{H} 3$ (UBI) $\mathrm{Ab}$ was added to the supernatant fraction and incubated overnight at $4{ }^{\circ} \mathrm{C}$ with rotation. Following washes and elution, histone-DNA cross-linked complexes were reversed by heating at $65^{\circ} \mathrm{C}$ for $4 \mathrm{~h}$. DNA was treated with EDTA, Tris HCL and proteinase K (UBI), extracted with phenol-chloroform, precipitated, and resuspended in $15 \mu 1$ of DEPC water. PCR detection of a 301-base HTLV-1 LTR DNA sequence was performed using the following primers: FWD 5'-CCGTCCTCAGGCGTTGA3' and REV 5'-CCGGCTGAGTCTAGGTAGGCT-3'.

Western blotting. Following TSA treatment, C81 cells were harvested, washed, and lysed in extraction buffer $(50 \mathrm{mM}$ Tris, $10 \%$ glycerol, $150 \mathrm{mM} \mathrm{NaCl}, \mathrm{pH} 8.0)$ by rocking at $4^{\circ} \mathrm{C}$ for $30 \mathrm{~min}$. Following centrifugation the whole cell extracts were removed and stored at $-80^{\circ} \mathrm{C}$ prior to use. Proteins were 


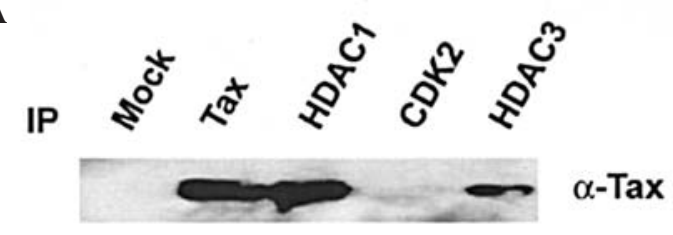

B

IP FLAG

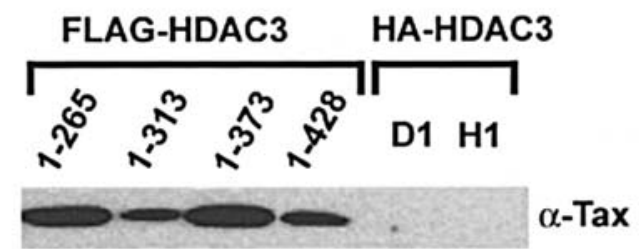

Figure 3. Immunoprecipitation of Tax and HDACs. (A) To examine whether endogenous HDACs and Tax physically associate in molecular complexes, we performed immunoprecipitation with Abs against Tax, HDACs 1 and 3 and CDK2, followed by Western blotting with anti-Tax antibodies. As shown, endogenous HDAC1 and HDAC3 but not CDK2 bind Tax in C81 cells. (B) C81 cell transfection with FLAG-HDAC3 constructs that encode proteins devoid of HDAC activity was performed for $48 \mathrm{~h}$, prior to whole cell extract isolation. Control HA-tagged D1 and $\mathrm{H} 1$ point mutant constructs were also transfected. IPs with anti-FLAG Abs followed by Western blotting with anti-Tax were then performed. The experiment reveals that all FLAG-tagged deletion constructs used in the study have preserved ability to bind Tax, suggesting that the region important for binding is localized in the $\mathrm{N}$-terminal half.

resolved by electrophoresis in 4-20\% gradient Tris-Glycine gel (NOVEX, San Diego, CA), and transferred to a nitrocellulose membrane filter (Millipore, Bedford, MA). Coomassie staining was used to assess sample loading. The membranes were blocked with $5 \%$ nonfat milk and probed with Abs against p40Tax (AIDS Research and Reference Reagent Program, NIH) at a dilution of 1:1,000. HRP-conjugated goat $\alpha$-rabbit secondary Ab (Affinity Bioreagents, Golden, CO) at a 1:10,000 dilution was used. The bands were visualized by the enhanced chemiluminescence system (NEN Life Science Products, Boston, MA) and exposure to X-ray film (Eastman Kodak, Rochester, NY). C81 cells were also transfected with WT HDAC3. From these cells, we isolated the acid-soluble fraction of whole proteins, using a histone isolation protocol recommended by the manufacturer, followed by Western blotting with $\alpha$-acetylated histone 3 (ac-H3) and 4 (ac-H4) Abs (UBI).

Transfections. The HDAC3 enzymatic point mutant constructs, D1 and H1, used in transfections, were generated in our laboratory (details provided upon request) with the QuikChange site-directed Mutagenesis kit (Stratagene), following the manufacturer's instructions. The D1 aspartic acid for asparagine (D168N) and $\mathrm{H} 1$ histidine for phenylalanine $(\mathrm{H} 135 \mathrm{~F})$ substitutions are both located deep in the tubular pocket that participates as the enzymatic site of HDAC3 and binding site for TSA (26). The D and $\mathrm{H}$ pocket residues are highly conserved throughout evolution, suggesting a critical function. Cells were transfected by electroporation with a Biorad electroporator ( $300 \mathrm{~V}$ and 1,000 microFarads). FLAG-tagged HDAC3 deletion mutants (1-265, 1-313 and 1-373) known to encode proteins deficient in HDAC activity (27), were kindly provided by Dr Edward Seto (University of South Florida).

Immunoprecipitation (IP). IP was performed as previously described (28). Briefly, proteins were recovered in Laemmli buffer after high speed 20-min centrifugation. IP was performed with Abs against HDAC1 (Affinity Bio-reagents), HDAC3 (SantaCruz Biotechnology), CDK2 (Clontech) and a mock (anti-HA) Ab (SantaCruz Biotech-nology) as follows: protein extracts were incubated overnight at $4^{\circ} \mathrm{C}$ with the $\mathrm{Ab}$, and immunoprecipitated proteins were recovered on protein A agarose (UBI) and washed for $5 \mathrm{~min} 3 \mathrm{x}$ at room temperature with $50 \mathrm{mM}$ Tris-HCL pH 8.0, $150 \mathrm{mM} \mathrm{NaCl}, 1$ mM EDTA and $0.1 \%$ NP-40. Gels were loaded with immunoprecipitates and run as described above and blots were probed with a primary $\mathrm{Ab}$ against Tax. In addition, IP with anti-FLAG Abs (Sigma) followed by Western blotting with anti-Tax Abs was performed in FLAG mutanttransfected C81 cells.

\section{Results}

We first treated C81 cells ( $1 \times 10^{6}$ per sample) for 8,12 and $24 \mathrm{~h}$ (Fig. 1A). TSA led to increased expression of Tax/Rex mRNA at 12 and $24 \mathrm{~h}$. To examine whether TSA-enhanced Tax mRNA expression was due to histone hyperacetylation at the HTLV-1 LTR and not due to nonspecific acetylation, we performed a parallel chromatin immunoprecipitation (ChIP) assay, as described in the Upstate Biotechnology protocol. As shown (Fig. 1A lower panel), TSA promotes local histone hyperacetylation at the HTLV-1 LTR as early as $8 \mathrm{~h}$, a time that precedes the upregulation of Tax mRNA.

We then examined whether TSA treatment led to elevation of the Tax protein in C81 cells, using Western blotting. Analysis of $\mathrm{C} 81$ protein extracts with antibodies against Tax revealed upregulation of Tax protein by TSA (Fig. 1B). To examine if the ability of TSA to enhance Tax mRNA expression was not exclusive to C81 cells, we examined expression in HTLV-1-infected T-cell clones by RT-PCR. TSA treatment resulted in upregulation of Tax mRNA in HTLV-1-infected Du43 cells (Fig. 1C).

To examine if HDAC3 plays a role in repressing Tax, we transfected C81 cells with full-length, wild-type (WT) HDAC3, a 428 amino acid-encoding cDNA that we and others cloned and characterized $(23,29,30)$, or the HDAC3 enzymatic site point mutants D1 and H1. Transfection with WT HDAC3 in a pCDNA3. $1 \mathrm{Zeo}^{+}$plasmid, as compared to vector alone, led to a dose-dependent decrease in Tax mRNA at $24 \mathrm{~h}$ (Fig. 2A). HDAC3 point mutants, D1 and H1, at a comparable dosage of $1 \mu \mathrm{g}$ DNA per transfection also repressed Tax but had a decreased ability to exert these effects when compared to WT HDAC3.

We then transfected cells with three FLAG-tagged HDAC3 deletion mutants (1-265, 1-313 and 1-373) known to encode proteins deficient in HDAC activity (27). Using these constructs, Yang et al have shown that the structural integrity 
of the C-terminus of HDAC3 is required for enzymatic and repressor activity. As shown in Fig. 2B, several regions of HDAC3 maintain repressive activity on Tax mRNA expression that approximate that of the wild-type (428 amino acid-encoding) molecule, and the repression is more evident with the inclusion of the C-terminal domains of HDAC3. Interestingly, the 1-373 construct is devoid of HDAC activity but is shown to have preserved ability to repress Tax. The results indicate that the repressor function of HDAC3 on Tax mRNA can occur independently of its enzymatic activity. To verify if HDAC3 (1-428) transfection correlated with histone deacetylation, we then transfected C81 cells with WT HDAC3. From these cells, we isolated the acid-soluble fraction of whole proteins, using a histone isolation protocol recommended by the manufacturer of $\alpha$-acetylated histone 3 (ac-H3) and 4 (ac-H4) Abs (UBI). Western blotting showed that, as compared to vector only-transfected cells, HDAC3 transfection led to decreased Tax protein expression that correlated with increased HDAC3 and decreased histone $\mathrm{H} 3$ and $\mathrm{H} 4$ acetylation (Fig. 2C).

To assess if Tax protein physically associates with HDAC proteins, we performed IPs. These experiments revealed that Tax is found complexed with HDAC1 or HDAC3 (Fig. 3A). To verify that the deletion mutants used in this study encoded proteins that retained the ability to bind Tax, we performed IPs on extracts from FLAG mutant-transfected C81 cells. IP with anti-FLAG Abs (Sigma) followed by Western blotting with anti-Tax Abs revealed that all the deletion constructs were capable of binding Tax (Fig. 3B). HA-tagged point mutant D1 and $\mathrm{H} 1$ constructs were used as negative IP controls for the anti-FLAG Abs.

\section{Discussion}

Lin et al (31) identified butyrate, a non-specific HDAC inhibitor, as a strong inducer of HTLV-1 RNA and Tax protein expression. HDAC inhibitors can upregulate expression of the feline foamy virus and other viruses in vitro $(32,33)$. In this study, we show that the specific HDAC inhibitor TSA led to upregulation of Tax mRNA in cells known to chronically harbor the HTLV-1 virus. Our demonstration of increased histone hyperacetylation at the HTLV-1 LTR site following TSA administration supports a direct effect of this drug on the HTLV-1 proviral genome.

Recent studies have shown that repressor complexes containing HDACs are associated with the 5' or 3' promoter HTLV-1 LTR in vivo (34). Such recruitment of HDACs to a retroviral promoter site has also been shown for HIV (35). HDAC1 binds the HTLV-1 Tax protein (36) and HDAC1 overexpression downregulates HTLV-1 Tax transcription $(34,37)$. Thus, Tax and HDAC1 may reciprocally inhibit each other's ability to interact with the HTLV-1 LTR $(34,36)$. Our study demonstrates that transfection with HDAC3 also downregulates HTLV-1 Tax expression, highlighting another layer of complexity for this repressor mechanism.

Further, we demonstrate a physical association between HDAC3 and Tax proteins. Our use of point mutants indicated that the ability of HDAC 3 to repress Tax may depend in part on the integrity of a region spanning the catalytic domain. This domain is composed of residues that contribute to the formation of the enzymatic pocket. The HDAC3 residues mutated in this study (amino acid residues 135 and 168) occupy deep sites of the tubular pocket of this enzyme $(26,38)$. However, the requirement for the structural integrity of the enzymatic region is not absolute, since enzymatically inactive HDAC3 fragments (27) remained capable of Tax repression. The region spanning amino acid residues 314-373 and 402-428 of HDAC3 had the strongest effects on Tax repression. The results suggest that other protein-protein interaction sites influence Tax repression independently of HDAC3's enzymatic function. These interactions may include recruitment of Tax to other sites of the HDAC3 protein, to enhance repression by physical proximity, or binding of other repressor molecules on the C-terminal half of the molecule. Of note, the Taxbinding domain of a closely related deacetylase, HDAC1, localizes between amino acid residues 29-97 of the N-terminus (37). All flagged HDAC3 constructs used for transfections in this study contained this $\mathrm{N}$-terminal region and their encoded proteins bound Tax protein, as assessed via immunoprecipitation studies.

Promoter DNA methylation is another silencing mechanism proposed to play a modulatory role in retroviral latency $(39,40)$. In fact, the HTLV-1 LTR has been shown to be highly methylated in Tax-nonexpressing cells derived from adult T-cell leukemia patients (40). In addition, HDACs bind DNA methyltransferases (DNMTs) (41), the chief enzymes responsible for DNA methylation. We have found that the DNA methylation inhibitor drug, 5-aza-2'deoxycytidine (AZA), also upregulated Tax expression (results not shown). Thus, the ability of TSA to induce Tax expression suggests that pharmacologic HDAC inhibition may simultaneously affect multiple, possibly convergent, gene silencing mechanisms. Our results support a role for HDAC3 in molecular complexes that mediate Tax function, and provide a basis for further exploration of retroviral latency mechanisms.

\section{Acknowledgments}

The authors would like to thank Francisca Beato and Cruz Martinez for technical advice and support. Rabbit $\alpha$-Tax Abs were obtained from the AIDS Research and Reference Reagent Program, Division of AIDS, NIAID, NIH, catalogue \#712 from Dr Kuan-Teh Jeang. This study was supported by NIH grant 1KO8CA80084-01A1

\section{References}

1. Kitajima I, Shinohara T, Bilakovics J, Brown DA, Xu X and Nerenberg M: Ablation of transplanted HTLV-I Taxtransformed tumors in mice by antisense inhibition of NF-kappa B. Science 258: 1792-1795, 1992.

2. Scherdin U, Rhodes K and Breindl M: Transcriptionally active genome regions are preferred targets for retrovirus integration. J Virol 64: 907-912, 1990.

3. Höllsberg P, Wucherpfennig KW, Ausubel LJ, Calvo V, Bierer BE and Hafler DA: Characterization of HTLV-I in vivo infected Tcell clones. IL-2- independent growth of nontransformed Tcells. J Immunol 148: 3256-3263, 1992.

4. Grassmann R, Berchtold S, Radant I, et al: Role of human T-cell leukemia virus type $1 \mathrm{X}$ region proteins in immortalization of primary human lymphocytes in culture. J Virol 66: 4570-4575, 1992.

5. Migone TS, Lin JX, Cereseto A, et al: Constitutively activated Jak-STAT pathway in T-cells transformed with HTLV-I. Science 269: 79-81, 1995. 
6. Xu X, Kang SH, Heidenreich O, Okerholm M, O'Shea JJ and Nerenberg MI: Constitutive activation of different Jak tyrosine kinases in human T-cell leukemia virus type 1 (HTLV-1) tax protein or virus-transformed cells. J Clin Invest 96: 1548-1555, 1995.

7. Azimi N, Mariner J, Jacobson S and Waldmann TA: How does interleukin 15 contribute to the pathogenesis of HTLV type 1associated myelopathy/tropical spastic paraparesis? AIDS Res Hum Retroviruses 16: 1717-1722, 2000.

8. Jeang KT: Functional activities of the human T-cell leukemia virus type I Tax oncoprotein: cellular signaling through NFkappa B. Cytokine Growth Factor Rev 12: 207-217, 2001

9. Lundblad JR, Kwok RP, Laurance ME, et al: The human T-cell leukemia virus-1 transcriptional activator Tax enhances cAMPresponsive element-binding protein (CREB) binding activity through interactions with the DNA minor groove. J Biol Chem 273: 19251-19259, 1998.

10. Lenzmeier BA, Giebler HA and Nyborg JK: Human T-cell leukemia virus type 1 Tax requires direct access to DNA for recruitment of CREB binding protein to the viral promoter. Mol Cell Biol 18: 721-731, 1998.

11. Sodroski JG, Rosen CA and Haseltine WA: Trans-acting transcriptional activation of the long terminal repeat of human T lymphotropic viruses in infected cells. Science 225: 381-385, 1984.

12. Paskalis H, Felber BK and Pavlakis GN: Cis-acting sequences responsible for the transcriptional activation of human T-cell leukemia virus type I constitute a conditional enhancer. Proc Natl Acad Sci USA 83: 6558-6562, 1986.

13. Brady J, Jeang KT, Duvall J and Khoury G: Identification of p40xresponsive regulatory sequences within the human T-cell leukemia virus type I long terminal repeat. J Virol 61: 2175-2181, 1987.

14. Montagne J, Beraud C, Crenon I, et al: Tax1 induction of the HTLV-I 21 bp enhancer requires cooperation between two cellular DNA-binding proteins. EMBO J 9: 957-964, 1990.

15. Bantignies F, Rousset R, Desbois C and Jalinot P: Genetic characterization of transactivation of the human T-cell leukemia virus type 1 promoter: Binding of Tax to Tax-responsive element 1 is mediated by the cyclic AMP-responsive members of the CREB/ATF family of transcription factors. Mol Cell Biol 16: 2174-2182, 1996.

16. Colgin MA and Nyborg JK: The human T-cell leukemia virus type 1 oncoprotein Tax inhibits the transcriptional activity of cMyb through competition for the CREB binding protein. J Virol 72: 9396-9399, 1998.

17. Harrod R, Tang Y, Nicot C, et al: An exposed KID-like domain in human T-cell lymphotropic virus type 1 Tax is responsible for the recruitment of coactivators $\mathrm{CBP} / \mathrm{p} 300$. Mol Cell Biol 18: 5052-5061, 1998

18. Yan JP, Garrus JE, Giebler HA, Stargell LA and Nyborg JK: Molecular interactions between the coactivator CBP and the human T-cell leukemia virus Tax protein. J Mol Biol 281: 395-400, 1998.

19. Bex F, Yin MJ, Burny A and Gaynor RB: Differential transcriptional activation by human T-cell leukemia virus type 1 Tax mutants is mediated by distinct interactions with CREB binding protein and p300. Mol Cell Biol 18: 2392-2405, 1998.

20. Kwok RP, Laurance ME, Lundblad JR, et al: Control of cAMPregulated enhancers by the viral transactivator Tax through CREB and the co-activator CBP. Nature 380: 642-646, 1996.

21. Jiang H, Lu H, Schiltz RL, et al: PCAF interacts with tax and stimulates tax transactivation in a histone acetyltransferaseindependent manner. Mol Cell Biol 19: 8136-8145, 1999.

22. Bannister AJ and Kouzarides T: The CBP co-activator is a histone acetyltransferase. Nature 384: 641-643, 1996.

23. Gray SG and Ekstrom TJ: The human histone deacetylase family. Exp Cell Res 262: 75-83, 2001.
24. Hebbes TR, Thorne AW and Crane-Robinson C: A direct link between core histone acetylation and transcriptionally active chromatin. EMBO J 7: 1395-1402, 1988.

25. Kinoshita T, Shimoyama M, Tobinai K, et al: Detection of mRNA for the tax1/rex1 gene of human T-cell leukemia virus type I in fresh peripheral blood mononuclear cells of adult Tcell leukemia patients and viral carriers by using the polymerase chain reaction. Proc Natl Acad Sci USA 86: 5620-5624, 1989

26. Finnin MS, Donigian JR, Cohen A, et al: Structures of a histone deacetylase homologue bound to the TSA and SAHA inhibitors. Nature 401: 188-193, 1999.

27. Yang WM, Tsai SC, Wen YD, Fejer G and Seto E: Functional domains of histone deacetylase-3. J Biol Chem 277: 9447-9454, 2002.

28. Iglesias AH, Camelo S, Hwang D, Villanueva R, Stephanopoulos G and Dangond F: Microarray detection of E2F pathway activation and other targets in multiple sclerosis peripheral blood mononuclear cells. J Neuroimmunol 150: 163-177, 2004.

29. Dangond F, Hafler DA, Tong JK, et al: Differential display cloning of a novel human histone deacetylase (HDAC3) cDNA from PHA-activated immune cells. Biochem Biophys Res Commun 242: 648-652, 1998.

30. Dangond F, Foerznler D, Weremowicz S, Morton CC, Beier DR and Gullans SR: Cloning and expression of a murine histone deacetylase 3 (mHdac3) cDNA and mapping to a region of conserved synteny between murine chromosome 18 and human chromosome 5. Mol Cell Biol Res Commun 2: 91-96, 1999.

31. Lin HC, Dezzutti CS, Lal RB and Rabson AB: Activation of human T-cell leukemia virus type 1 tax gene expression in chronically infected T-cells. J Virol 72: 6264-6270, 1998.

32. Gray SG and Teh BT: Histone Acetylation/Deacetylation and cancer: An "open" and "shut" case? Curr Mol Med 1: 401-429, 2001.

33. Hatama S, Otake K, Ohta M, et al: Reactivation of feline foamy virus from a chronically infected feline renal cell line by trichostatin A. Virology 283: 315-323, 2001.

34. Lemasson I, Polakowski NJ, Laybourn PJ and Nyborg JK: Transcription regulatory complexes bind the human T-cell leukemia virus 5 ' and 3 ' long terminal repeats to control gene expression. Mol Cell Biol 24: 6117-6126, 2004.

35. Coull JJ, Romerio F, Sun JM, et al: The human factors YY1 and LSF repress the human immunodeficiency virus type 1 long terminal repeat via recruitment of histone deacetylase 1. J Virol 74: 6790-6799, 2000.

36. Lu H, Pise-Masison CA, Linton R, et al: Tax relieves transcriptional repression by promoting histone deacetylase 1 release from the human T-cell leukemia virus type 1 long terminal repeat. J Virol 78: 6735-6743, 2004

37. Ego T, Ariumi Y and Shimotohno K: The interaction of HTLV1 Tax with HDAC1 negatively regulates the viral gene expression. Oncogene 21: 7241-7246, 2002.

38. Hassig CA, Tong JK, Fleischer TC, et al: A role for histone deacetylase activity in HDAC1-mediated transcriptional repression. Proc Natl Acad Sci USA 95: 3519-3524, 1998.

39. Koiwa T, Hamano-Usami A, Ishida T, et al: 5'-long terminal repeat-selective $\mathrm{CpG}$ methylation of latent human $\mathrm{T}$-cell leukemia virus type 1 provirus in vitro and in vivo. J Virol 76: 9389-9397, 2002.

40. Saggioro D, Panozzo M and Chieco-Bianchi L: Human Tlymphotropic virus type I transcriptional regulation by methylation. Cancer Res 50: 4968-4973, 1990.

41. Fuks F, Burgers WA, Brehm A, Hughes-Davies L and Kouzarides T: DNA methyltransferase Dnmt1 associates with histone deacetylase activity. Nat Genet 24: 88-91, 2000. 